\title{
Combined effects of physical inactivity and sedentary behaviour on psychological distress among university-based young adults: A one-year prospective study
}

\author{
Riaz Uddin ${ }^{1, *}$, Nicola W Burton ${ }^{2,3}$ and Asaduzzaman Khan ${ }^{1}$
}

${ }^{1}$ School of Health and Rehabilitation Sciences, The University of Queensland, Australia

${ }^{2}$ School of Applied Psychology, Griffith University, Australia

${ }^{3}$ School of Human Movement and Nutrition Sciences, The University of Queensland, Australia

${ }^{*}$ Corresponding author (RU)

The University of Queensland School of Health and Rehabilitation Sciences Brisbane, QLD 4072, Australia

Phone: +61 414217 225; Fax: +61 733651877

Ethics approval: All procedures performed in studies involving human participants were in accordance with the ethical standards of the institutional and/or national research committee and with the 1964 Helsinki declaration and its later amendments or comparable ethical standards. The University of Queensland Behavioural and Social Sciences Ethical Review Committee, Australia provided ethical approval for the study (Ref: 2015000860; Amendment- 31/07/2015; Amendment 29/09/2016). Informed consent was obtained from all individual participants included in the study.

Acknowledgements: This research was supported by an Australian Government Research Training Program Scholarship awarded to RU. The authors would like to thank the participating students, and the class lecturers who helped access the students at their classroom. The authors also would like to acknowledge the support of the authorities of the participating institutions for allowing their students to participate in the survey. The second author (NWB) was with The University of Queensland School of Human Movement and Nutrition Sciences when the study was conceived, designed and data were collected.

Competing interests: None to declare.

Funding source: This research did not receive any specific grant from funding agencies in the public, commercial, or not-for-profit sectors.

Citation: Uddin R, Burton NW, Khan A. Combined effects of physical inactivity and sedentary behaviour on psychological distress among university-based young adults: A one year prospective study. Psychiatric Quarterly. Published online December 6, 2019. doi: https://doi.org/10.1007/s11126-019-09697-2 


\title{
Combined effects of physical inactivity and sedentary behaviour on psychological distress among university-based young adults: A one-year prospective study
}

\author{
Abstract \\ Insufficient physical activity (PA) and prolonged sedentary behaviour (SB) may have deleterious \\ psychological health consequences. Using one-year prospective data, this study examined the \\ combined effects of PA and SB on psychological distress among university-based young adults in \\ Dhaka, Bangladesh. During Wave 1 (September to December 2015), total 573 undergraduate \\ students (average age: $20.7 \pm 1.35$ years; $45 \%$ female) completed a self-administered survey on PA, \\ SB, psychological distress, health and lifestyle factors and sociodemographics. During Wave 2 \\ (October-November 2016), 395 students (retention rate=69\%) completed a sub-set of Wave 2 \\ survey with items on PA, SB and psychological distress. PA and SB were assessed using the Global \\ Physical Activity Questionnaire (GPAQ) and distress with the Kessler 6 Psychological Distress \\ (K6) scale. Generalized Estimating Equations (GEE) with Gaussian family and identity link under \\ exchangeable correlation structure was used to examine the relationships. Multivariable modelling \\ showed that participants with insufficient PA $(<150$ minutes/week) and high SB $(\geq 480$ \\ minutes/day) or insufficient PA + low SB had more distress [3: 3.07 (95\%CI: 2.12-4.01) and 2.77 \\ (1.86-3.67), respectively] than those who had sufficient PA + low SB, after controlling for gender, \\ sleep difficulties, perceived health, fast food and fresh fruit intake. There was no statistically \\ significant difference for the level of distress between other PA and SB groups. In this one-year \\ prospective study, irrespective of $\mathrm{SB}$, insufficient PA was associated with high psychological \\ distress in university-based young adults in Bangladesh. The protective role of PA should be \\ considered in intervention programs to improve the psychological health of young adults.
}

Keywords: exercise; health promotion; longitudinal study; mental health; screen use 


\section{Introduction}

Approximately three-quarters of psychological health problems appear by the age of 20 years [1]. Worldwide, poor psychological health has been reported as the most common cause of disability in adolescents and young adults aged 10-24 years, contributing to $45 \%$ of years lost due to disability (YLD) [2]. Globally, unipolar depressive disorders are the leading cause of non-fatal burden of disease in young adults aged 20-24 years, representing approximately $8 \%$ of total disabilityadjusted life-years (DALYs) lost [2]. Poor psychological health may compromise psychosocial development, increase substance abuse, lower academic performance and achievements, cause unemployment and decrease the quality of life [3-6].

Physical activity (PA) participation has been inversely associated with general young adults' poor psychological health in longitudinal [7] and intervention studies [8-10]. PA can reduce the risk of anxiety and depressive symptoms, and stress; and improve the psychosocial wellbeing of young adults [7-10]. In contrast, sedentary behaviour (SB), defined as waking time spent in sitting or reclining; e.g., watching television (TV), computer use, in travel (e.g., in motorised vehicles), has been positively associated with poor psychological health in young adults $[11,12]$. For example, a recent longitudinal study in Chinese college students reported that high screen-time was associated with poor psychological health, and an increase in screen-time was associated with progression of psychological health problems during one-year follow-up [12].

Insufficient PA and high SB may interact to increase psychological health problems [13-17]. For example, a recent cross-sectional study in Chinese college students reported that participants who had insufficient PA ( $<3$ days/week of exercise) and high SB ( $>2$ hours/day screen-time) were more likely to report depression, anxiety, and other psychopathological symptoms than those who had sufficient PA and low SB [16]. However, the strength of this evidence is limited by a crosssectional study design. 
University students may be vulnerable to poor psychological health because of academic and social demands in their day-to-day life [6]. Epidemiological data from around the world suggest a high prevalence of depression and anxiety in this population with $12 \%-50 \%$ meeting criteria for at least one common psychological disorders [6, 5]. Insufficient PA and high level of SB are also common in university-based young adults, and may be associated with poor psychological wellbeing $[14,12,16]$. This study aimed to use one-year prospective data to examine the associations of PA and SB with psychological distress in a university-based sample of young adults in Dhaka City, Bangladesh.

\section{Methods}

\section{Study design and population}

Data are from a one-year prospective study: Wave 1 (September to December 2015) and Wave 2 (October-November 2016). A convenience sample of first, second and third-year students aged 18 to 24 years was recruited from six (three public and three private) universities based in Dhaka City, Bangladesh. The recruitment procedure can be found elsewhere [18]. Fourth-year students were not included at Wave 1 as they were unlikely to be available for follow-up.

Data were collected using a self-administered survey, which was administered in English. Completion of Wave 1 survey took approximately 40-45 minutes, with questions about PA, SB, psychological distress, health and lifestyle factors, and socio-demographics. Wave 2 survey was a sub-set of Wave 1 survey including items on PA, SB, and psychological distress; this took about 15-20 minutes to complete.

\section{Outcome measure - psychological distress}

The Kessler Psychological Distress (K6) scale has been shown to be a reliable and validated selfreport to assess general psychological health in a community setting [19]. The K6 scale, consisting of six items, coded from 0 to $4(0=$ 'none of the time' to $4=$ 'all of the time'), asks how frequently 
in the past four weeks respondents had experienced each of the following commonly occurring symptoms: sadness, nervousness, fidgety, hopelessness, effortfulness and worthlessness. The six items were added to generate a total score (range: 0-24) with high scores representing more distress. An exploratory factor analysis with varimax rotation confirmed the unidimensionality of the scale in the current study with a one-factor solution, which accounted for $59 \%$ and $62 \%$ of the variance respectively during Wave 1 and Wave 2. Cronbach's alpha for the scale in this study was 0.85 at Wave 1 and 0.82 at Wave 2.

\section{Other measures}

PA and SB were measured using the Global Physical Activity Questionnaire (GPAQ), which was developed by the World Health Organisation (WHO) for population surveillance in low- and middle-income countries [20]. Reliability and validity of the GPAQ have been assessed among adults aged $\geq 18$ years in nine countries, including Bangladesh [20]. It has been used in $>100$ countries around the world, including Bangladesh, in the STEPwise Approach to Noncommunicable Disease Risk-Factor Surveillance (STEPS) program by the WHO [20]. The current study used the self-administered version, which is a relatively inexpensive method with comparable reliability and validity to the interview administration [21]. It consists of 15 items on PA and one on SB [20].

\section{Physical activity}

The respondents were asked to report the number of days in a week, and the amount of time in a typical day of the week, they spent doing each of moderate and vigorous PA for work and recreation; and moderate PA for walking or cycling for travel. Consistent with the GPAQ data analysis guideline, all activity data were converted to minutes and were multiplied by the corresponding number of days [22]. Vigorous activity minutes were weighted by two given the higher intensity than moderate activity [22]. The time spent in vigorous activity (weighted) and moderate activity minutes were summed to obtain total PA, which was then dichotomised as 
'sufficient $\mathrm{PA}$ ' ( $\geq 150$ minutes/week) and 'insufficient PA' ( $<150$ minutes/week). This is consistent with the WHO's PA recommendations [22]. As per the GPAQ protocol, four respondents (two at Wave 1 and two at Wave 2) were excluded from the analyses as they provided improbable or out of range PA/SB data during [22]. This has been described in details elsewhere [18].

\section{Sedentary behaviour}

The GPAQ asks the participant to report total time spent sitting/reclining (excluding sleeping) during a typical day of the week [20]. As young adults are more sedentary on weekend days than weekdays [23], the single question was split into two for a typical weekday and weekend day using the same response options. Total sedentary time (mins/day) was derived as:

Daily sedentary time $($ mins $)=[($ weekday time $\times 5)+($ weekend day time $\times 2)] / 2$

Currently, there is no consensus threshold at which a person to be defined to have high SB. However, sitting for $\geq 8 \mathrm{hrs} /$ day (480 mins/day) has been shown to significantly increase the risk of all-cause mortality [24]. This value was therefore used in the present study with SB dichotomised as 'low' ( $<480 \mathrm{~min} /$ day) and 'high', ( $\geq 480 \mathrm{~min} /$ day). This criterion has also been used in other research in Asian adults [25].

\section{Socio-demographics and lifestyle factors}

Participants completed survey items to assess: age, gender, height and weight, marital status (married/unmarried), university type (public/private), enrolled program (Science; Humanities; Engineering; and Business), year of study (year 1 to 3), parents' education level (primary or equivalent; secondary or equivalent; higher-secondary or equivalent; and tertiary or equivalent), mothers' current occupation (working/non-working), fathers' current occupation (government or public service; private sector; professional; self-employed/business; and other, which included semi-skilled and unskilled labour, and farmers), monthly gross household income $[\leq 20,000$ Bangladeshi Taka (BDT); 20,001-40,000; 40,001-70,000; >70,000], household composition (living 
alone; with parents; with family members other than parents; with friends/classmates), and housing type (own house/flat with family; rented house/flat with family; university accommodation/hall; shared accommodation with friends/classmates). Body mass index (BMI) was computed from selfreported height and weight, which was used as a continuous variable. Participants reported on cigarette smoking (non-smoker, past smoker, occasional smoker, regular smoker); alcohol consumption (I never drink, used to drink previously, drink occasionally, drink regularly); dietary behaviour (e.g., number of servings of each of fast food, fresh fruit, vegetables/green salad during last seven days); frequency of consumption of nonalcoholic carbonated beverages during the past seven days (none, 1-2, 3-4, 5-6, 7-9, 10-13, 14+ glass/cans), and if they had a TV in the bedroom (yes/no). Participants were also asked to rate current health (with five response categories collapsed into poor/fair and good/very good/excellent) and the frequency of experiencing sleep difficulties in the past month (with five response categories collapsed into always/often, sometimes, and rarely/never). Poor health and sleep difficulties have been associated with insufficient PA and poor psychological health in previous research [26, 14].

\section{Statistical analyses}

Dichotomous PA (sufficient and insufficient) and SB (high and low) variables were used to create a $2 \times 2$ variable of combinations of $\mathrm{PA}$ and $\mathrm{SB}$ : sufficient $\mathrm{PA}+$ low $\mathrm{SB}$; sufficient $\mathrm{PA}+$ high $\mathrm{SB}$; insufficient PA+low SB; and insufficient PA+high SB. The outcome variable (K6 score) was normally distributed, hence the Generalized Estimating Equations (GEE) with Gaussian family and identity link under exchangeable correlation structure was used to examine combined effects of PA and SB with the total K6 scores. In this analysis, GEE took into account the non-independence of students' psychological distress nested within two waves. The following potential covariates assessed at Wave 1 were considered as potential confounders: age, gender, marital status, BMI, parental education and occupation, monthly gross household income, household composition, housing type, television (TV) in bedroom, perceived health, sleep difficulties, smoking, alcohol consumption, fast food intake, fresh fruit and vegetable intake, nonalcoholic carbonated beverage 
intake, university type, enrolled program, and year of study. Potential confounding variables which had univariate associations with the K6 score at $\mathrm{p}<.20$ [as recommended elsewhere [27]] were identified and examined for collinearity. BMI, mother's education, father's occupation, household composition and housing type were initially considered in the multivariable model, but were not significantly associated with the K6 score in the multivariable model and were excluded. Gender, sleep difficulties, perceived health, fast food and fresh fruit intake were significantly associated with the K6 score in the final multivariable GEE model.

A number of sensitivity analysis were conducted: a) unlike PA, there is no established cutoff value for high SB. Hence, three different cut-offs were used to define high SB ( $\geq 420 \mathrm{~min} /$ day, $\geq$ $540 \mathrm{~min} /$ day, and $\geq 600 \mathrm{~min} /$ day) to examine the impact of categorisation on results; b) a complete case analysis with only completers those provided valid data during both assessment waves was run to examine possible bias from dropouts; and c) the associations were examined using multilevel generalised structural equation modelling (SEM) to see if such modelling changed the results. Multilevel generalised SEM took into account the nesting of the participants across the two waves.

Outliers and other assumptions of the models were checked and model fit was assessed before finalising the models. The associations are presented in the form of regression coefficients with their 95\% confidence intervals. All analyses were performed in STATA version 14 (StataCorp LP., College Station, Texas) with statistical significance set at $\mathrm{p}<.05$.

\section{Results}

Of the 573 students (response rate: 91.6\%) who participated in Wave 1 survey, 397 completed the Wave 2 survey (retention rate: $68.6 \%$ ). Two respondents were excluded as they provided incomplete data - thus, the analytical sample of this study is 395. At baseline, the average age of the participants was 20.7 years $(\mathrm{SD}=1.35)$ and $45 \%$ were female. Table 1 summarises the baseline characteristics of the participants. 
The sociodemographic characteristics of Wave 2 respondents were comparable to Wave 1 participants for gender (females $45 \%$ vs $48 \%$ during Wave 1 and Wave 2, respectively), BMI $\left(21.6 \pm 3.3 \mathrm{~kg} / \mathrm{m}^{2}\right.$ vs $\left.22.1 \pm 2.9 \mathrm{~kg} / \mathrm{m}^{2}\right)$, being single (94\% vs $\left.92 \%\right)$, living with parents (47\% vs $\left.52 \%\right)$ and type of university (public $48 \%$ vs $42 \%$ ). 
Table 1 Baseline characteristics of the participating university-based young adults in Dhaka City, Bangladesh ( $\mathrm{N}=573), 2015$

\begin{tabular}{|c|c|c|c|}
\hline \multicolumn{2}{|c|}{ Characteristics } & $\mathbf{n}^{\mathbf{z}}$ & $\%$ \\
\hline \multicolumn{4}{|c|}{ Age (years) } \\
\hline & Mean $20.7(\mathrm{SD}=1.35)$ & - & - \\
\hline \multicolumn{4}{|l|}{ Gender } \\
\hline & Male & 313 & 54.6 \\
\hline & Female & 260 & 45.4 \\
\hline \multicolumn{4}{|c|}{$\operatorname{BMI}\left(\mathrm{kg} / \mathrm{m}^{2}\right)$} \\
\hline & Normal range & 353 & 61.7 \\
\hline & Underweight & 139 & 24.3 \\
\hline & Overweight & 80 & 14.0 \\
\hline \multicolumn{4}{|c|}{ University type } \\
\hline & Public & 277 & 48.3 \\
\hline & Private & 296 & 51.7 \\
\hline \multicolumn{4}{|c|}{ Year of study } \\
\hline & First year & 184 & 32.1 \\
\hline & Second year & 223 & 38.9 \\
\hline & Third year & 166 & 29.0 \\
\hline \multicolumn{4}{|c|}{ Mother's educational qualification } \\
\hline & Primary or equivalent & 111 & 19.4 \\
\hline & Secondary (or equivalent) & 147 & 25.7 \\
\hline & Higher secondary (or equivalent) & 125 & 21.9 \\
\hline & Tertiary (or equivalent) & 188 & 32.9 \\
\hline \multicolumn{4}{|c|}{ Father's educational qualification } \\
\hline & Primary or equivalent & 53 & 9.3 \\
\hline & Secondary (or equivalent) & 64 & 11.2 \\
\hline & Higher secondary (or equivalent) & 102 & 17.9 \\
\hline & Tertiary (or equivalent) & 352 & 61.7 \\
\hline \multicolumn{4}{|c|}{ Monthly gross family income (in BDT) ${ }^{*}$} \\
\hline & $\leq 20,000$ & 115 & 20.3 \\
\hline & $20,001-40,000$ & 162 & 28.6 \\
\hline & $40,001-70,000$ & 172 & 30.4 \\
\hline & $>70,000$ & 117 & 20.7 \\
\hline \multicolumn{4}{|c|}{ Household composition } \\
\hline & Living alone & 24 & 4.2 \\
\hline & Living with parents & 270 & 47.2 \\
\hline & Living with family members other than parents & 46 & 8.4 \\
\hline & Living with friends/classmates & 232 & 40.6 \\
\hline \multicolumn{4}{|c|}{ Housing type } \\
\hline & Own house/flat with family & 181 & 31.6 \\
\hline & Rented house/flat with family & 176 & 30.7 \\
\hline & University accommodation/hall & 146 & 25.5 \\
\hline & Shared accommodation with friends/classmates & 70 & 12.2 \\
\hline
\end{tabular}

${ }^{¥}$ Total for each variable may not be equal to $n=573$ due to missing values 
After adjusting for gender, sleep difficulties, perceived health, fast food and fresh fruit intake, GEE modelling showed that participants with insufficient PA+high SB had a significantly higher level of psychological distress than those who had sufficient PA+low SB [adjusted ß: 3.07, 95\% CI: 2.12-4.01, p<.001]. Participants who had insufficient PA+low SB also reported more psychological distress than young adults did with sufficient PA+low SB [2.77 (1.90-3.71), $\mathrm{p}<.001]$ after adjusting for the confounders. There was no statistically significant difference for the level of psychological distress between participants with sufficient $\mathrm{PA}+$ high $\mathrm{SB}$, and those with sufficient $\mathrm{PA}+$ low SB $(\mathrm{p}=.362)$ (Table 2).

Table 2 Associations of physical activity and sedentary behaviour with psychological distress scores of university-based young adults in Dhaka City, Bangladesh (N=395), 2015/16

\begin{tabular}{|l|l|l|l|l|}
\hline PA and SB categorise & \multicolumn{2}{|l|}{ Unadjusted estimates } & \multicolumn{2}{l|}{ Adjusted estimates* } \\
\hline & $\beta(95 \% \mathrm{CI})$ & $p$-value & $\beta(95 \% \mathrm{CI})$ & $p$-value \\
\hline Sufficient PA + low SB & Reference & & Reference & \\
\hline Sufficient PA + high SB & -0.67 & .322 & -0.59 & .362 \\
\hline Insufficient PA + low SB & $(-2.00$ to 0.66$)$ & & $(-1.86$ to 0.68$)$ & \\
\hline Insufficient PA + high SB & 3.42 & $<.001$ & 2.77 & $<.001$ \\
& $(2.51$ to 4.34$)$ & & $(1.86$ to 3.67$)$ & \\
\hline & $(2.97$ to 4.86$)$ & & $(2.12-4.01)$ & \\
\hline
\end{tabular}

Values in bold indicate p-value of 0.05 or lower.

${ }^{\dagger}$ Estimates are based on Generalized Estimating Equations (GEE) with the K6 psychological distress scores as dependent variable

*Adjusted for gender, sleep difficulties, perceived health, frequency of fast food and fresh fruit consumption Abbreviations: $\mathrm{CI}=$ confidence intervals; $\mathrm{PA}=$ physical activity; $\mathrm{SB}=$ sedentary behaviour

Additionally, there was no statistically significant difference for the level of psychological distress between participants with insufficient PA+high SB, and those with 
insufficient PA+low SB ( $\mathrm{p}=.336$ ). However, compared to sufficient PA+high SB, participants with insufficient PA+high SB [3.66 (2.50-4.81); $\mathrm{p}<.001]$, and insufficient PA+low SB [3.36 $(2.23-4.48) ; \mathrm{p}<.001]$ had a higher level of psychological distress (results are not shown in the table) after adjusting for the confounders. High SB was not independently associated with psychological distress $(\mathrm{p}=.638)$ after adjusting for the same set of confounders (results are not shown in the table).

Results from the sensitivity analysis on GEE platform using different cut-off values for SB did not change the overall results, which suggests that SB categorisation did not affect the results. Findings of the complete case analysis with those provided data on both assessment points did not influence the results; and therefore suggests that findings are not influenced by dropouts. Findings of the multilevel generalised SEM did not change the results suggesting stability of the findings across different modelling platforms.

\section{Discussion}

The main finding of this one-year prospective study is that insufficient PA (i.e., $>150$ mins/week) was associated with high psychological distress irrespective of SB, after controlling for a set of potential confounders. Findings suggest that insufficient PA and high SB increased psychological distress significantly compared to sufficient PA and low SB in the study participants. This is understandable given other research indicating the deleterious effects of each of low PA and high SB [8, 9, 28, 11, 10, 12]. Insufficient PA and low SB also significantly increased psychological distress, which is also consistent with an expected adverse impact of low PA. However, there were no significant differences in distress between sufficient PA and high SB, and sufficient PA and low SB. This is inconsistent with other research demonstrating adverse effects of high SB [12] and suggests that PA may provide protective effects or have a stronger association with psychological health than SB. 
Our findings are consistent with a previous cross-sectional study with adolescents that reported insufficient PA increased depressive symptomology, though the combination of insufficient PA and high SB did not [29]. These findings, however, are inconsistent with previous cross-sectional studies reporting higher odds of anxiety, depression and other psychopathological problems in university students with high SB and insufficient PA than those with low SB and sufficient PA $[14,12]$. The inconsistency may be due to the different measures used to examine the relationships. For example, the study in Chinese college students which found that students with insufficient PA and high SB had poorer psychological health than those who had sufficient PA and low SB used the Self-rating Anxiety Scale, Centre for Epidemiologic Studies Depression Scale and Multidimensional Sub-health Questionnaire for Adolescents [16], and this study used a brief composite measure of general psychological distress. The other study used frequency of participation as a measure of PA [16], whereas this study used time spent in PA. Sedentary behaviour was assessed as time spent using a computer and watching TV/video in the other study [16] and a global measure of time spent sitting/reclining that could have included behaviours other than screen time was used in this current study. A recent meta-analysis of 101 studies found no relationship between video gaming and psychological health problems in youth [30]. Thus, it is possible that not all types of SB are associated with poor psychological health, and that the association depends on the contexts of SB. Future research could examine how different types of SB might interact with PA to influence the psychological health of young adults.

Findings of this study suggest that insufficient PA has significant negative effects on psychological health of university-based young adults, regardless of SB. The underlying mechanism by which PA influences psychological health may involve biological, psychosocial, and/or common cause mechanisms [31]. Regular PA has a beneficial effect on the hypothalamic-pituitary-adrenal (HPA) system. Dysregulation of the HPA-axis and 
hypersecretion of cortisol may have an adverse effect on psychological health [32]. PA may act as a moderator to activate the limbic system and reduce cortisol secretion level [33]. Insufficient PA may interfere with the HPA functioning system, change serum cortisol level, and cause psychological distress. Dysregulation of the HPA system and sympathetic adrenal medullary, which is caused by insufficient PA may also be responsible for the cardiometabolic syndrome [34] and increase metabolic disease risk [35], which have been linked to poor psychological health [34]. It is also plausible that insufficient PA may interfere with a number of growth factors such as brain-derived neurotrophins and insulin-like growth factor, which are enhanced by exercise, and offer therapeutic and protective effects against depression [32]. PA may augment brain aminergic synaptic transmission of monoamines such as norepinephrine, dopamine and serotonin and may also activate $\beta$-endorphin secretion, which may subsequently improve psychological health [36].

Psychosocial factors may also explain the relationship. Regular PA may promote selfesteem, a positive body image and health perceptions [37], increase social support, positive emotions [38] and overall life satisfaction [39]. Insufficient PA may have a negative impact on psychosocial resources such as social support and purpose in life [38], and may increase depression and anxiety [40, 41].

It is possible that the relationship between poor psychological health and PA is bidirectional [42]. To explore the possibility of bi-directionality, additional modelling was conducted considering each of PA and $\mathrm{SB}$ as dependent variable and $\mathrm{K} 6$ scores as an independent variable. The results demonstrated that psychological distress was significantly associated with insufficient PA; however, the association was not significant with SB (results not presented). While insufficient PA may have an adverse effect on psychological health, it is also possible that poor psychological health negatively influences PA participation [42, 
31]. For example, depression may negatively influence PA through a range of affective symptoms such as low mood and sadness, anhedonia, and low self-esteem, and somatic symptoms such as lethargy [31].

The relationship between insufficient PA and poor psychological health may also be explained by common cause hypothesis [31]. For example, insufficient PA and depression may share a range of factors including genetic makeup, socioeconomic status, the neighbourhood where an individual resides and parenteral rearing style [31, 43], which may influence both PA and psychological health. It is possible that PA participants are, in general, more satisfied with their lives and are happier than non-PA participants [43].

To our knowledge, this is the first study that examined the combined effects of PA and SB on psychological health in young adults using prospective data. This study used PA and SB measures, which have been validated in Bangladeshi adults, and adjusted the relationships for multiple covariates including sleep difficulties and subjective health of the participants to minimise possible confounding. A moderately large, heterogeneous sample of students from both public and private universities with a balanced representation of both sexes was used. This study followed-up the participants over one year with two assessment points, which did not allow examination of how the effect(s) of PA and SB on psychological health evolves over the long term. Self-reported measures were used to assess PA, SB and psychological distress, which are vulnerable to social desirability and recall bias. Though the K6 scale has good reliability and validity in other countries, it has not been validated in a Bangladeshi population. As a non-random convenience sample of university students from a metropolitan city was used, findings may have limited generalisability, and may not be transferable to all young adults. 


\section{Conclusions}

In this one-year prospective study, insufficient PA was associated with high psychological distress irrespective of SB in a university-based young adult population in Dhaka City, Bangladesh. Our findings reinforce the importance of PA participation during young adulthood to mitigate possible psychological health risks. Our study findings do not suggest any unique combined effect of insufficient PA and low SB on the psychological health of young adults in urban Bangladesh. More long-term prospective studies are needed to further investigate the independent and interactive effects of PA and different types of SB on psychological health to advance our current understanding of these relationships.

\section{References}

1. World Health Organization. Adolescents and mental health. n.d. http://www.who.int/maternal_child_adolescent/topics/adolescence/mental_health/en/. Accessed $12 / 06 / 2017$.

2. Gore FM, Bloem PJN, Patton GC, Ferguson J, Joseph V, Coffey C et al. Global burden of disease in young people aged 10-24 years: a systematic analysis. Lancet. 2011;377(9783):2093-102. doi:10.1016/S0140-6736(11)60512-6.

3. Gibb SJ, Fergusson DM, Horwood LJ. Burden of psychiatric disorder in young adulthood and life outcomes at age 30. British Journal of Psychiatry. 2010;197(2):122-7. doi:10.1192/bjp.bp.109.076570.

4. Patel V, Flisher AJ, Hetrick S, McGorry P. Mental health of young people: a global public-health challenge. Lancet. 2007;369(9569):1302-13. doi:10.1016/S0140-6736(07)60368-7.

5. Bruffaerts R, Mortier P, Kiekens G, Auerbach RP, Cuijpers P, Demyttenaere K et al. Mental health problems in college freshmen: Prevalence and academic functioning. Journal of Affective Disorders.225:97-103. doi:10.1016/j.jad.2017.07.044.

6. Bayram N, Bilgel N. The prevalence and socio-demographic correlations of depression, anxiety and stress among a group of university students. Social Psychiatry and Psychiatric Epidemiology. 2008;43(8):667-72. doi:10.1007/s00127-008-0345-x.

7. Ströhle A, Höfler M, Pfister H, Müller A-G, Hoyer J, Wittchen H-U et al. Physical activity and prevalence and incidence of mental disorders in adolescents and young adults. Psychological Medicine. 2007;37(11):1657-66. doi:doi:10.1017/S003329170700089X.

8. Al-Eisa E, Buragadda S, Melam GR. Association between physical activity and psychological status among Saudi female students. BMC Psychiatry. 2014;14(1):238. doi:10.1186/s12888-0140238-3. 
9. Ghorbani F, Heidarimoghadam R, Karami M, Fathi K, Minasian V, Bahram ME. The effect of sixweek aerobic training program on cardiovascular fitness, body composition and mental health among female students. Journal of Research in Health Sciences. 2014;14(4):264-7.

10. Li M, Fang Q, Li J, Zheng X, Tao J, Yan X et al. The effect of Chinese traditional ExerciseBaduanjin on physical and psychological well-being of college students: A randomized controlled trial. PLoS One. 2015;10(7):e0130544. doi:10.1371/journal.pone.0130544.

11. Edwards MK, Loprinzi PD. Effects of a sedentary behavior-inducing randomized controlled intervention on depression and mood profile in active young adults. Mayo Clinic Proceedings. 2016;91(8):984-98. doi:10.1016/j.mayocp.2016.03.021.

12. Wu X, Tao S, Zhang S, Zhang Y, Chen K, Yang Y et al. Impact of screen time on mental health problems progression in youth: a 1-year follow-up study. BMJ Open. 2016;6(11):e011533. doi:10.1136/bmjopen-2016-011533).

13. Cao H, Qian Q, Weng T, Yuan C, Sun Y, Wang H et al. Screen time, physical activity and mental health among urban adolescents in China. Preventive Medicine. 2011;53(4-5):316-20. doi:10.1016/j.ypmed.2011.09.002.

14. Feng Q, Zhang Q-1, Du Y, Ye Y-1, He Q-q. Associations of physical activity, screen time with depression, anxiety and sleep quality among Chinese college freshmen. PLoS One. 2014;9(6):e100914. doi:10.1371/journal.pone.0100914.

15. Khan A, Burton NW. Is physical inactivity associated with depressive symptoms among adolescents with high screen time? Evidence from a developing country. Mental Health and Physical Activity. 2017;12:94-9. doi:10.1016/j.mhpa.2017.03.001.

16. Wu X, Tao S, Zhang Y, Zhang S, Tao F. Low physical activity and high screen time can increase the risks of mental health problems and poor sleep quality among Chinese college students. PLoS One. 2015;10(3):e0119607. doi:10.1371/journal.pone.0119607.

17. Khan A, Uddin R, Burton NW. Insufficient physical activity in combination with high screen time is associated with adolescents' psychosocial difficulties. International Health. 2018. doi:10.1093/inthealth/ihy019.

18. Uddin R, Khan A, Burton NW. Prevalence and sociodemographic patterns of physical activity among Bangladeshi young adults. Journal of Health, Population and Nutrition. 2017;36(1):31. doi:10.1186/s41043-017-0108-y.

19. Kessler RC, Andrews G, Colpe LJ, Hiripi E, Mroczek DK, Normand SL et al. Short screening scales to monitor population prevalences and trends in non-specific psychological distress. Psychological Medicine. 2002;32(6):959-76. doi:10.1017/S0033291702006074.

20. Bull FC, Maslin TS, Armstrong T. Global physical activity questionnaire (GPAQ): nine country reliability and validity study. Journal of Physical Activity and Health. 2009;6:790-804.

21. Chu AH, Ng SH, Koh D, Muller-Riemenschneider F. Reliability and validity of the self- and interviewer-administered versions of the Global Physical Activity Questionnaire (GPAQ). PLoS One. 2015;10(9):e0136944. doi:10.1371/journal.pone.0136944.

22. World Health Organization. Global Physical Activity Questionnaire (GPAQ) Analysis Guide. Geneva, Switzerland: World Health Organization2012.

23. Arias-Palencia NM, Solera-Martínez M, Gracia-Marco L, Silva P, Martínez-Vizcaíno V, CañeteGarcía-Prieto $J$ et al. Levels and patterns of objectively assessed physical activity and compliance with different public health guidelines in university students. PLoS One. 2015;10(11):e0141977. doi:10.1371/journal.pone.0141977.

24. Chau JY, Grunseit A, Midthjell K, Holmen J, Holmen TL, Bauman AE et al. Sedentary behaviour and risk of mortality from all-causes and cardiometabolic diseases in adults: evidence from the 
HUNT3 population cohort. British Journal of Sports Medicine. 2015;49(11):737-42. doi:10.1136/bjsports-2012-091974.

25. Win AM, Yen LW, Tan KH, Lim RBT, Chia KS, Mueller-Riemenschneider F. Patterns of physical activity and sedentary behavior in a representative sample of a multi-ethnic South-East Asian population: a cross-sectional study. BMC Public Health. 2015;15(1):318. doi:10.1186/s12889-015$1668-7$.

26. Hoyt LT, Chase-Lansdale PL, McDade TW, Adam EK. Positive youth, healthy adults: Does positive well-being in adolescence predict better perceived health and fewer risky health behaviors in young adulthood? Journal of Adolescent Health. 2012;50(1):66-73.

doi:10.1016/j.jadohealth.2011.05.002.

27. Maldonado G, Greenland S. Simulation study of confounder-selection strategies. American Journal of Epidemiology. 1993;138(11):923-36. doi:10.1093/oxfordjournals.aje.a116813.

28. Ströhle A. Physical activity, exercise, depression and anxiety disorders. Journal of Neural Transmission. 2009;116(6):777-84. doi:10.1007/s00702-008-0092-x.

29. Kremer P, Elshaug C, Leslie E, Toumbourou JW, Patton GC, Williams J. Physical activity, leisure-time screen use and depression among children and young adolescents. Journal of Science and Medicine in Sport. 2014;17(2):183-7. doi:10.1016/j.jsams.2013.03.012.

30. Ferguson CJ. Do angry birds make for angry children? A meta-analysis of video game influences on children's and adolescents' aggression, mental health, prosocial behavior, and academic performance. Perspectives on Psychological Science. 2015;10(5):646-66.

doi:10.1177/1745691615592234.

31. Stavrakakis N, de Jonge P, Ormel J, Oldehinkel AJ. Bidirectional prospective associations between physical activity and depressive symptoms. The TRAILS Study. Journal of Adolescent Health. 2012;50(5):503-8. doi:doi:10.1016/j.jadohealth.2011.09.004.

32. Hamer M. Psychosocial stress and cardiovascular disease risk: the role of physical activity. Psychosomatic Medicine. 2012;74(9):896-903. doi:10.1097/PSY.0b013e31827457f4.

33. Nabkasorn C, Miyai N, Sootmongkol A, Junprasert S, Yamamoto H, Arita M et al. Effects of physical exercise on depression, neuroendocrine stress hormones and physiological fitness in adolescent females with depressive symptoms. European Journal of Public Health. 2006;16(2):17984. doi:doi:10.1093/eurpub/cki159.

34. Goldbacher EM, Matthews KA. Are psychological characteristics related to risk of the metabolic syndrome? A review of the literature. Annals of Behavioral Medicine. 2007;34(3):240-52. doi:10.1007/BF02874549.

35. Wijndaele K, Brage S, Besson H, Khaw K-T, Sharp SJ, Luben R et al. Television viewing time independently predicts all-cause and cardiovascular mortality: the EPIC Norfolk study. International Journal of Epidemiology. 2010;40(1):150-9. doi:doi:10.1093/ije/dyq105.

36. Paluska SA, Schwenk TL. Physical activity and mental health: current concepts. Sports Medicine. 2000;29(3):167-80. doi:10.2165/00007256-200029030-00003.

37. Korn L, Gonen E, Shaked Y, Golan M. Health perceptions, self and body image, physical activity and nutrition among undergraduate students in Israel. PLoS One. 2013;8(3):e58543. doi:10.1371/journal.pone.0058543.

38. Hogan CL, Catalino LI, Mata J, Fredrickson BL. Beyond emotional benefits: Physical activity and sedentary behaviour affect psychosocial resources through emotions. Psychology \& Health. 2015;30(3):354-69. doi:10.1080/08870446.2014.973410.

39. Rangul V, Bauman A, Holmen TL, Midthjell K. Is physical activity maintenance from adolescence to young adulthood associated with reduced CVD risk factors, improved mental health 
and satisfaction with life: the HUNT Study, Norway. International Journal of Behavioral Nutrition and Physical Activity. 2012;9:144-. doi:10.1186/1479-5868-9-144.

40. Martinsen EW. Physical activity in the prevention and treatment of anxiety and depression. Nordic Journal of Psychiatry. 2008;62(sup47):25-9. doi:10.1080/08039480802315640.

41. Teychenne M, Abbott G, Lamb KE, Rosenbaum S, Ball K. Is the link between movement and mental health a two-way street? Prospective associations between physical activity, sedentary behaviour and depressive symptoms among women living in socioeconomically disadvantaged neighbourhoods. Preventive Medicine. 2017;102:72-8. doi:10.1016/j.ypmed.2017.07.005.

42. Gunnell KE, Flament MF, Buchholz A, Henderson KA, Obeid N, Schubert N et al. Examining the bidirectional relationship between physical activity, screen time, and symptoms of anxiety and depression over time during adolescence. Preventive Medicine. 2016;88:147-52. doi:10.1016/j.ypmed.2016.04.002.

43. Stubbe JH, De Moor MHM, Boomsma DI, De Geus EJC. The association between exercise participation and well-being: a co-twin study. Preventive Medicine. 2007;44(2):148-52. doi:10.1016/j.ypmed.2006.09.002. 